\title{
SPECULATIONS CONCERNING POSSIBLE NEW PHYSICS FROM EXPERIMENTS ABOVE $10 \mathrm{TeV}^{*}$
}

\author{
G.I. Kane \\ Randall Laboratory of Physics \\ University of Michigan \\ Ann Arbor, MI 48109
}

We can classify the kinds of new physics which we can hope to learn from experiments at higher energies -from cosmic rays and from colliding beam accelerators -into two categories.

(A) Standard experiments. These include a number of important areas which have been well studied and reviewed already, such as (a) measurement of $\sigma_{\text {TOT' }}$ scaling and scaling violations, and related "lns" physics; (b) production of gauge bosons $\mathrm{w}^{ \pm}, \mathrm{z}^{\circ} ;$ and (c) large $\mathrm{p}_{\mathrm{T}}$ data resulting from basic constituent interactions. Since these have been well covered I will on the whole not discuss them here.

(B) Other. There are a number of possible new kinds of effects. Of course, one could speculate without constraint, but today one has available viable gauge theories of strong, weak, and electromagnetic interactions and it seems preferable to speculate within the context of these new effects. It may be that none of the following speculations will be realistic and we are only free to discuss them at the moment because our understanding of the theory is minimal. Even so, some good, either theoretical or experimental, may come from such considerations.

The topics I will discuss are

(1) Possible large "weak interactions" at very high energies, with an associated cross section that is a significant part of $\sigma_{\text {TOT }}$; what characteristics would enable us to detect such new effects?

(2) Can we learn some things about QCD and gluons from small $\mathrm{p}_{\mathrm{T}}$, large cross section experiments?

tResearch supported in part by the U.S.D.O.E. 
(3) At large $p_{T}$ but very high energy, cross sections in $Q C D$ get very large.

(4) Some new heavy meson excitations are suggested by increased understanding of the gauge theories and could appear experimentally.

\section{LARGE "WEAK INTERACTIONS" AT VERY HIGH ENERGIES}

What happens at very high energies in the unified gauge theories of weak and electromagnetic interactions? First, consider the Weinberg-Salam theory, (1) with a single Higgs boson doublet to provide the masses of the gauge bosons $\left(\mathrm{W}^{ \pm}, \mathrm{Z}^{\circ}\right)$ and the fermions, via spontaneous symmetry breaking. Suppose the Higgs boson masses are not large, i.e. $\mathrm{m}_{\mathrm{H}} \leqslant \mathrm{m}_{\mathrm{W}}$. Then $i t$ can be shown that no weak interaction effects get large. At very large $p_{T}$, for example, the weak and electromagnetic cross sections will be comparable in size. The weak interaction will be relatively isotropic, so it is a tiny part of the cross section at small $\mathrm{p}_{\mathrm{T}}$, and thus a tiny part altogether.

However, if $\mathrm{m}_{\mathrm{H}}>>\mathrm{m}$, some amplitudes in the boson sector become larger $(2,3)$, because the coupling strengths are proportional to $\mathrm{m}_{\mathrm{H}}$. Then one can imagine obtaining large cross sections. It is interesting to consider ways to observe possible "weak" effects at very high energies. Before we do that, briefly consider how such effects could be imagined to arise.

Consider Higgs boson scattering via an s-channel Higgs,

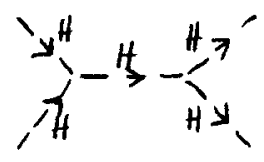

giving an amplitude

$$
M=\frac{9}{4} g^{2} \frac{m_{H}^{2}}{m_{W}^{2}} \frac{m_{H}^{2}}{s-m_{H}^{2}}=18 \quad\left(G_{F} m_{H}^{2}\right) m_{H}^{2} /\left(s-m_{H}^{2}\right)
$$

and a cross section

$$
\sigma=\frac{81}{4 \pi s}\left(G_{F} m_{H}^{2}\right)^{2} \frac{\left(m^{2} H\right)^{2}}{\left(s-m_{H}^{2}\right)^{2}} .
$$


If we assume $m_{H}=1 \mathrm{TeV}=\sqrt{\mathrm{s}}$, an energy which can be achieved by the colliding doubler at FNAL even for constituent subenergies, and put $s-m_{H}^{2} \simeq m_{H^{T}}{ }^{\prime}$, then $\sigma \simeq 260 \mathrm{mb} / \Gamma_{\mathrm{H}}^{2}$ with $\Gamma_{\mathrm{H}}$ in $\mathrm{GeV}$. Such a cross section greatly exceeds the unitarity limit, since it arises from a single partial wave. similar results arise for other diagrams. Consequently they will be suppressed. Nevertheless, it is conceivable that the dynamics gives rise to amplitudes from the Higgs sector which are large enough to give observable effects, directly or through interferences with strong interactions.

Is this relevant to experiments? Here a subtle complication arises -- in the simplest Weinberg-Salam theory, with one Higgs doublet, even if large effects did arise the answer is no. $(2,4)$ To do experiments one must begin with fermions; e.g. qq or $\mathrm{e}^{+} \mathrm{e}^{-}$collide (the $q \bar{q}$ in hadrons). Then all of the large amplitudes get multiplied by the tiny factor $\left(\mathrm{m}_{\mathrm{f}} / \mathrm{m}_{\mathrm{W}}\right)^{2}$, essentially because that factor appears in the Higgs-fermion coupling. one can make a strong statement: in the standard theory at high energies there will be no weak interaction effects not directly due to production of $W^{\prime} ' s$ and $Z$ 's; observation of such effects would contradict the standard theory.

Is this general? No. One can construct theories $(5,6,7,8)$ which differ only in the Higgs sector, in which the Higgs-fermion coupling is no longer suppressed by the factor $\mathrm{m}_{\mathrm{f}} / \mathrm{m}_{\mathrm{W}}$. In essence one needs two Higgs doublets, one having vacuum expectation value $v$ and giving mass to fermions, with $v \sim m_{f}$, and the other having vacuum expectation value $V$ and giving mass to the gauge bosons, with $\mathrm{V} \approx \mathrm{m}_{\mathrm{W}}$. No contradiction with experiment arises (8) for Higgs-fermion couplings up to about two orders of magnitude times that of the standard theory. While an extension of the standard theory along these lines is not required by any data, it is of interest to have the boson and fermion mass scales set separately in the Higgs sector. If nature were like this extension it would be relatively easy to detect Higgs bosons and their effects, and - most relevant here -- it is not prohibited from giving large cross sections associated with the Higgs sector at high energies. 
So far I have argued that even within the context of the conventional gauge theories it could happen that large cross sections arise from effects associated with the Higgs sector. The arguments are meant to stimulate our thinking rather than to lead to definite conclusions; they certainly do not imply that such effects will be present. More important is the question: how could we detect large Higgsrelated phenomena if they were present?

To give some answers to this, consider the characteristics of normal strong interactions at present experimental energies:

(a) Hadrons have a certain size, and differential and total cross sections are qualitatively fixed by geometrical arguments, $\sigma_{\text {TOT }} \simeq \pi R^{2}, d \sigma / d t \simeq \sigma_{\text {TOT }}^{2} \exp \left(R^{2} t\right)$ $\mathrm{do} / \mathrm{dt}$ also has a diffraction minimum.

(b) Isospin is a good symmetry

(c) Parity is a good symmetry

(d) Large $p_{T}$ events are very improbable

(e) Increased energy input goes mainly into relative motion, not into particle masses and increased multiplicity. Consequently, multiplicity grows as $n$ s.

If a new interaction (e.g. related to the Higgs sector as above) becomes important at very high energies there is no guarantee that it will respect any of these results. Consider them in turn.

(a) If a new interaction is present it could have a distance scale which is not connected to
normal hadronic sizes. Then $\sigma_{\text {TOT }}$ could change fairly abruptly to a new value; presumably only an increase will occur as a different distribution in partial waves would be involved and cancellations are unlikely. Similarly, do/dt could broaden significantly if a part of the cross section involved scattering on a shorter distance scale. (In this context it is amusing that recent data ${ }^{(10)}$ shows a relatively isotropic do/dt for pp elastic scattering at $400 \mathrm{GeV} / \mathrm{c}$, with $\mathrm{d} \sigma / d t \sim \exp (0.7 \mathrm{t})$ for $-t \simeq 10 \mathrm{GeV}^{2}$, while for small $t, d \sigma / d t \sim \exp (12 t)$.

(b) Since Higgs-quark couplings are proportional to the quark mass, and ${ }^{(11)} \mathrm{m}_{\mathrm{u}} \neq \mathrm{m}_{\mathrm{d}}$, the Higgs-quark interaction does not conserve isospin. If it led to large high energy interactions, one might find 
large isospin violations at such energies. For example, one could find

$$
\sigma_{\mathrm{TOT}}(\mathrm{pp}) \neq \sigma_{\mathrm{TOT}}(\mathrm{np}) \neq \sigma_{\mathrm{TOT}}(\mathrm{pd}) / 2
$$

or

$$
\mathrm{n}\left(\pi^{0}\right) \neq\left(\mathrm{n}\left(\pi^{+}\right)+\mathrm{n}\left(\pi^{-}\right)\right) / 2
$$

with similar results for other resonances. At present there is no way to estimate what effects to expect (if any); the key point is that such effects could occur and we should be alert to find them.

(c) In a similar way it could happen that large parity-violation effects occur at high energies. For example, to give an illustrative mechanism, in the model of Ref. 8 the charged Higgs can be quite massive and it couples only to left-handed fermions. There are several ways ${ }^{(12)}$ to detect such effects. distribution

-- If a $\Lambda$ is detected, it has a decay angular

$$
\begin{aligned}
W_{A}(\theta, \phi) & =N\left\{I+\alpha \sin \theta \sin \phi P_{Y}+\alpha \sin \theta \cos \phi P_{X}\right. \\
& \left.+\alpha \cos \theta P_{z}\right\},
\end{aligned}
$$

where $\theta, \phi$ are the decay angles in the $\Lambda$ rest frame, $\mathrm{P}_{\mathrm{X}, \mathrm{y}, \mathrm{z}}$ the components of the $\Lambda$ polarization, and $\alpha$ the measured asymmetry, $\alpha \simeq 2 / 3$. If parity is conserved in the production of the $\Lambda$, then $\mathrm{P}_{\mathrm{x}}=\mathrm{P}_{\mathrm{z}}=0$. Thus the presence of the $\sin \theta \cos \phi$ or $\cos \theta$ terms would require parity violation in the production. A non-zero $P_{z}$ could occur from direct "weak" production of the $\Lambda$, while a non-zero $P_{x}$ would have to arise from interference of a parity-violating and a parity-conserving amplitude of non-zero radative phase. Similar results (12) hold for production of $\phi, \mathrm{K}^{*}, \rho, \Delta, \ldots$ In general it is possible to learn from the strong decay angular distribution of a resonance if its production was parity violating. such as -- One can form parity-violating observables 


$$
\left\langle\overrightarrow{\mathrm{p}}_{\pi^{+}} \times \overrightarrow{\mathrm{p}}_{\pi^{-}} \cdot \overrightarrow{\mathrm{p}}_{\text {beam }}\right\rangle
$$

and look for non-zero values.

-- Consider a large $p_{T}$ quark jet, which fragments into two $\left(\pi_{a}, \pi_{b}\right)$ or more pions. Then the angular correlations of $\vec{p}_{a}, \vec{p}_{b}$ may allow the determination of longitudinal and transverse polarizations of $q$.

(d) A new mechanism associated with Higgs or "weak" interactions could presumably lead to different behavior at large $p_{T}$. This could get confused with the changes in large $p_{T}$ behavior expected from $Q C D$ when hard scattering of constituents takes over (see below), and it will be important not only to look for changes at large $p_{T}$ but to check whether the usual strong interaction symmetry laws apply, as expected in QCD.

(e) Finally, if a new mechanism enters there is no reason for the energy dependence of the multiplicity to stay the same. There could also be different multiplicity growth in different regions. Of course, it could happen that accidently two different mechanisms gave the same growth, or, multiplicity changes could be an easily detected clue to new phenomena.

How likely is any of the above scenarios? Although they are plausible, presumably they are not very likely. But the important thing is that the absence of any new effects at very high energies would also tell us a great deal about how the theory develops. Since good theories exist now, it is of great value to confirm their predictions, and to help guide their application in areas where calculations are difficult.

$$
\text { Low } \mathrm{p}_{\mathrm{T}} \mathrm{QCD}
$$

Next turn to possible ways of learning about gluons and QCD from small $\mathrm{p}_{\mathrm{T}}$ information. This is not as good as looking directly at perturbative $Q C D$ arguments and experiments directly related to $q q$ or $q g$ collisions. However, there may be useful physics to be learned, and a good deal of the total cross section is relevant 
to this region so it merits serious study. $(14,15)$. I'll mention three possibilities.

(a) It may be that single gluons give rise to the central region clusters. $(14,15)$ An important test of this is their flavor neutrality if they originate solely from gluons. Theoretically, it will eventally be possible to calculate multiplicities, mass distributions, rapidity gap distributions in terms of QCD quantities. These will have some s-dependence -- at higher energy, are there more clusters with energy-independent characteristics, or do the characteristics change too?

(b) An important possibility may be the opportunity to directly measure the gluon distribution function in the most important region of $x$, around $x \simeq 1 / 3$. To see this, consider particle ratios in central region production. since hadrons from gluons will be flavor neutral, ratios such as $\pi^{+} / \pi^{-}, \mathrm{K}^{+} / \mathrm{K}^{-}, \overline{\mathrm{p}} / \mathrm{p}, \bar{\Lambda} / \Lambda$ must go to one if the hadrons come from gluons.

Normally it is very hard to measure the gluon distribution function, i.e. the probability of finding gluons with a fraction $x$ of the hadrons momentum $G(x)$, because weak and electromagnetic currents do not interact with gluons. Consider the curves in Figure 2,

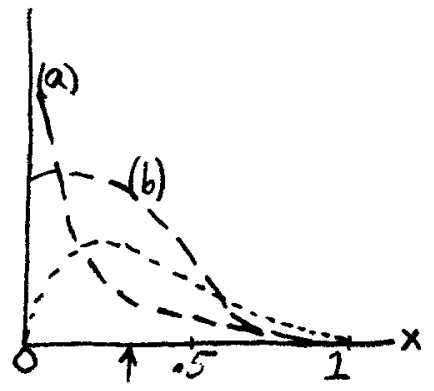

where the dashed lines show two possible gluon functions (a), and (b), and the dotted line the valence quark distribution. Look at $x \simeq 0.3$ (the arrow). Then for distribution (a) the quarks dominate over the gluons, and the particle ratios will not be unity, nor will they get closer to unity as $s$ increases and the gluons have more opportunity to dominate the central region. But for distribution (b), the gluons dominate over the quarks, and as $\mathbf{s}$ increases the particle ratios will get closer to unity at this $x$ value. At each $x$, we can determine whether $G(x)>g(x), G(x) \simeq q(x)$, or $G(x) \ll q(x)$. 
Since $q(x)$ is measured in lepton reactions, and the area under $G(x)$ is known from the momentum sum rule, this effectively allows a useful determination of $G(x)$. Data at current energies suggests that such a procedure may work at higher energies.

(c) An important prediction of $Q C D$ is that gluongluon interactions will be strong, and that probably resonant states -- called glue-balls -- will be formed. Although this subject is not well understood ${ }^{(16)}$ most work indicates that $\sigma_{g g}$ will be large, with several lowspin, low-mass resonances. Perhaps it will look as in Figure 3, where the dashed Iine indicates the unitarity limit.

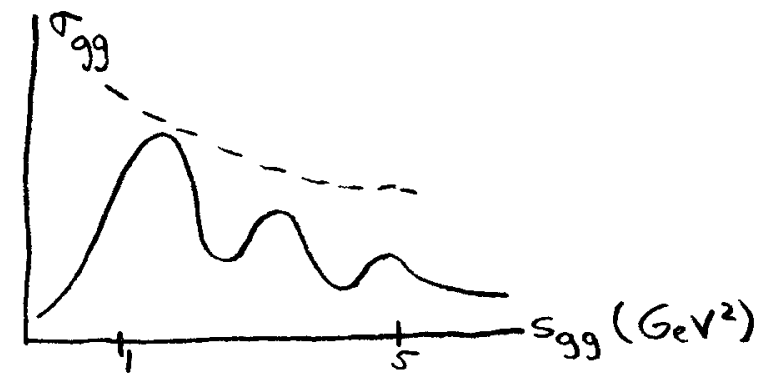

Solutions to QCD will have definite predictions for $\sigma_{\mathrm{gg}}$ as well as the gluon distribution functions. One possible indirect way to probe these is via the behavior of the very high energy total cross section. If we assume part of $\sigma_{\text {TOT }}$ is due to gluon-gluon interactions, we can write that part as

$$
\Delta \sigma_{\text {TOT }}^{G}=\int d x_{1} d x_{2} G\left(x_{1}\right) G\left(x_{2}\right) \sigma_{g g}\left(x_{1} x_{2} s\right)
$$

Where $G\left(x_{1}\right)$ gives the probability of finding one gluon with momentum fraction $x_{1}$ in one hadron, and similarly for $G\left(x_{2}\right)$. One can argue $(15)$ that the observed rise in $\sigma_{\text {TOT }}$ is due to such a mechanism. For example, at low energies and typical $x$ values, ${ }^{\sigma} g g$ is small. At higher energies $\sigma_{g g}$ is in the region of $i$ ts unitarity limit and $\Delta \sigma G$ TOT is large. If $G(x) \sim 1 / x$ for small $x$, as is expected, then $\Delta \sigma_{\text {TOT }}^{\mathrm{G}}$ grows as $\mathrm{lns}\left(\mathrm{\ell n}^{2} \mathrm{~s}\right)$ if $\sigma_{\mathrm{gg}}$ falls (becomes constant) at large $s$. The absolute size calculated from the above formula is consistent with what is needed to 
explain ISR data, assuming a $\sigma_{\text {gg }}$ with a few low energy resonances. Thus eventually, as we understood $Q C D$ better, it may be possible to predict the size and s-dependence of $\sigma_{\text {TOT }}$ at very high energies in terms of low energy gluon properties.

\section{LARGE $P_{T} Q C D$}

Finally I will give a few remarks from a conventional large $p_{T}$ QCD viewpoint. These are based on calculations from R. Field ${ }^{(17)}$. The main point is that at very high energies $Q C D$ predicts that the large $p_{T}$ cross section will be rather large. For example, the inclusive $\pi^{\circ}$ cross section has the property

$$
\begin{aligned}
E \frac{d \sigma}{d^{3} p} & \left(\pi^{\circ}, p_{T}=30 \mathrm{GeV} / \mathrm{c}, 90^{\circ}, \sqrt{\mathrm{s}}=500 \mathrm{GeV}\right) \\
\simeq & E \frac{\mathrm{d} \sigma}{\mathrm{d}^{3} \mathrm{p}}\left(\pi^{\circ}, \mathrm{p}_{\mathrm{T}}=6,90^{\circ}, \sqrt{\mathrm{s}}=19.4\right) .
\end{aligned}
$$

Similarly, the cross section for $\mathrm{pp} \rightarrow$ a jet with $\mathrm{p}_{\mathrm{T}}>2$ GeV has risen from of order $1 \%$ of $\sigma_{\text {TOT }}$ at (say) $\sqrt{5}=15$ $\mathrm{GeV}$, to as much as 408 of $\sigma_{\text {TOT }}$ for $\sqrt{\mathrm{s}}=800 \mathrm{GeV}$.

These cross sections are large because the cross section grows and approaches $a p_{T}^{-4}$ fall off as the energy increase. This is due in part to the asymptotically free nature of the theory. It is also kinematic in part, with interactions occuring at parton $x$ values more toward the maxima of the parton distributons as more energy is available and the kinematic restrictions matter less.

It will be of great importance to check these rather dramatic predictions.

\section{HEAVY MESONS}

Just as glueballs arise from the interactions of the quantum in $Q C D$, analagous excitations will be found in Weinberg-Salam theory. Recently, Nambu(18) has shown that classical solutions representing monopoleantimonopole configurations may be stable or nearly so and may give particle or resonance solutions. Einhorn and Savit have shown (19) that stable configurations of $\mathrm{Z}^{\circ}$-flux may exist as particle states $\left(\mathrm{Z}^{\circ}-\mathrm{balls}\right)$. These 
could decay into several $z^{\circ}$ 's plus whatever states $z^{\circ}$ is strongly coupled to, such as fermion-antifermion pairs.

So far, the arguments used by the above authors have been made for classical solutions, which correspond to highly excited states. However dimensional arguments alone indicate that the masses would be large, of order $1 \mathrm{TeV}$. Whether production cross sections will be large enough to lead to observation of such states is not known, but is not excluded. Both theoretically and experimentally, the existence of such states in the spectrum of gauge theories must be taken seriously.

\section{CONCLUSIONS}

Even within the framework of the conventional gauge theories it could happen that rather surprising behavior is in store at very high energies, including significant changes in $\sigma_{\text {TOT }}$ and $\mathrm{d \sigma} / \mathrm{dt}$, and large parity or isospin violations. On the other hand, perhaps no new kinds of effects occur. The latter alternative should not be considered boring; it has implications for the development of the gauge theories that are just as interesting and important as if some dramatic new behavior is found.

\section{ACKNOWLEDGMENTS}

I would like to thank the organizers, especially T. Gaisser, for their hospitality and for the opportunity to speak on this subject, and M. Einhorn for helpful conversations.

\section{REFERENCES}

1. S. Weinberg, Phys. Rev. Lett. 19, (1967) 1264; A. Salam, in Elementary Particle Physics, ed. by N. Svartholm. (Almquist and Wiksells, Stockholm 1968).

2. M. Veltman, Acta Physica Polonica B8, (1977) 475.

3. B.W. Lee, C. Quigg, and H.B. Thacker, Phys. Rev. D16, (1977) 1519.

4. H. Haber and G.I. Kane, Nuc. Phys. B, in press; UM HE 78-19.

5. F. Wilczek, Phys. Rev. Lett. 39, (1977) 1304.

6. J.E. Kim and G. Segre, University of Penn. Preprint UPR-0094T.

7. P. Ramond and G.G. Ross, Cal Tech preprint LACT-68-674.

8. H.E. Haber, G.L. Kane, and T. Sterling, preprint UM HE 78-45.

9. Through dispersion relations there will be constraints on how rapidly and how much $\sigma_{\text {TOT }}$ can chiange. These

have been considered by Pomeranchuk. See, for example Nuc. Sci. Abs. 30490; "Bounds on the Range 
of Increase of Weak Interaction Cross Sections", Pomeranchuk, I. Ya., Yadem. Fiz:, 11, 852 (1970).

10. S. Conetti et al., Phys. Rev. Lett. 41, 924 (1978).

11. S. Weinberg, "The Problem of Mass", A Festschrift in honor of I.I. Rabi, (N.Y. Acad of Sc., 1977).

12. H.E. Haber and G.L. Kane, Nuc. Phys. B, inpress; UM HE 78-11.

13. G.I. Kane, J. Pumplin, and W. Repko, UM HE 78-29, submitted to Phys. Rev. Lett.

14. L. Van Hove and S. Pokorski, Nuc. Phys. B86, (1975) 243; L. Van Hove, Acta Phys. Pol. B7, (1976) 339.

15. G.L. Kane and Y.P. Yao, Nuc. Phys. B137, (1978) 313

16. For a recent summary of work, see G.L. Kane, Proc. of the XIII Annual Rencontre de Moriond, March 1978.

17. R. Field, Private communication, and invited plenary session talk at the XIX International Conference on High Energy Physics, Tokyo, 1978, CALT-68-683.

18. Y. Nambu, Nucl. Phys. B130, 505 (1977).

19. M.B. Einhorn and R. Savit, Phys. Letters 77B, 295 (1978). 